\title{
Progress on Design of Emulsion Delivery System for Insoluble Bioactive Substances
}

\author{
Xueyan $\mathrm{Li}^{a}{ }^{a}$ Junwen Wang, ${ }^{a}$ and Chaoyan Zhang ${ }^{*, a, b, c, d}$ \\ ${ }^{a}$ Department of Marine Bio-Pharmacology, College of Food Science and Technology, \\ Shanghai Ocean University, Shanghai 201306, China \\ ${ }^{b}$ National Freshwater Aquatic Products Processing Technology R\&D Center (Shanghai), \\ Shanghai 201306, China \\ ${ }^{c}$ Shanghai Aquatic Products Processing and Storage Engineering Technology Research Center, \\ Shanghai 201306, China \\ ${ }^{d}$ Laboratory of Storage and Preservation Quality and Safety Risk Assessment of Aquatic Products \\ of the Ministry of Agriculture (Shanghai), Shanghai 201306, China
}

Email: cyzhang@shou.edu.cn (C. Z.)

\begin{abstract}
The appropriate delivery system is necessary for insoluble bioactive substances with poor water solubility, instability and low bioavaiability. The delivery system is key to improve its application characteristics. In terms of improving bioavailability of insoluble substances, emulsions have great advantages over other delivery systems. In this paper, the present researches on emulsion carrier systems of insoluble substances are reviewed including carotenoids, anthraquinones, diketone compounds, flavonoids, etc. At the same time, a summary on the development of insoluble substances food and drugs is presented, which is beneficial to the improvement of the research technology in this field.
\end{abstract}

Keywords emulsion delivery system, insoluble substances, stability, bioavailability

\section{Introduction}

The demand of bioactive substances used in food and drugs to enhance their health care function and efficacy is increasing due to their advantages such as antioxidant, anti-inflammatory, anti-tumor, and so on. However, water solubility of insoluble bioactive substances is poor, causing low oral bioavailability. ${ }^{[1]}$ After entering the gastrointestinal tract, the amount of bioactive substances absorbed into the blood circulation through gastrointestinal tract is limited, and most of them are vulnerable to damage by environmental factors. Therefore, improving the solubility and bioavailability of insoluble bioactive substances is urgent. Carrier system is the main way to solve the bioavailability, solubility and stability of insoluble bioactive substances. More than $40 \%$ of the newly discovered chemicals are poor in water solubility and low in oral bioavailability. In recent years, nano-emulsion as a tool for the efficient transmission of these low bioavailability molecules is receiving more and more attention. ${ }^{[2]}$

Nanosystems such as microemulsion and nano-emulsion are stable colloidal systems composed of oil and water, stabilized by a mixture of surfactants and cosurfactants. ${ }^{[3]}$ The particle size of nano-emulsion is $10-100 \mathrm{~nm}$, with high optical clarity, blue or light blue, low viscosity, isotropic thermodynamic and kinetic stable transparent or translucent system. ${ }^{[4,5]}$ Submicron emulsion is an isotropic mixture of drugs, lipids and surfactants. It usually has hydrophilic cosolvent and the droplet diameter is between 10 and $500 \mathrm{~nm}$. The submicron emulsion has good kinetic stability, high solubility and small spherical size ${ }^{[6]}$ Emulsion, microemulsion and nano-emulsion can transport active molecules, improve their solubility and stability, and reduce the side effects to a minimum (Table 1). ${ }^{[7]}$
Table 1 System composition of different emulsions

\begin{tabular}{ccc}
\hline $\begin{array}{c}\text { Emulsion } \\
\text { category }\end{array}$ & Grain size & System \\
\hline Nano-emulsion & $10-100 \mathrm{~nm}$ & $\begin{array}{c}\text { Carrier oil, emulsifier, } \\
\text { surfactants, water } \\
\text { Carrier oil, emulsifier, } \\
\text { surfactants, water } \\
\text { Micron emulsion }\end{array}$ \\
Self & $<500 \mathrm{~nm}$ & $\begin{array}{c}\text { Oil phase, surface } \\
\text { active agent, } \\
\text { cosurfactant }\end{array}$ \\
\hline
\end{tabular}

\section{Carotenoids}

Carotenoid is a natural fat-soluble pigment, which is obtained by chemical synthesis or extraction from plants, photosynthetic bacteria and microalgae. Generally speaking, these carotenoids are all e configuration, which have the characteristics of high crystallinity and low solubility. Because of its potential to prevent various diseases such as cancer and arteriosclerosis as well as its strong antioxidant capacity, it attracted extensive attention. ${ }^{[8]}$ Taking astaxanthin as an example, the effects of solubility and bioavailability of astaxanthin on emulsion delivery system were introduced.

Astaxanthin is an important carotenoid, and the special long-chain co-branched olefin structure in the molecular structure is endowed with the function of effectively quenching the active oxygen, has three isomeric forms of $3 S-3$ ' $S, 3 R-3$ ' $S$, $3 R-3 ' R$ (also referred to as left-hand, meso, and right-hand) (Figure 1). It is widely used in fish such as bacteria, algae, yeast, plant, crustacean and salmon. ${ }^{[9]}$ The astaxanthin 


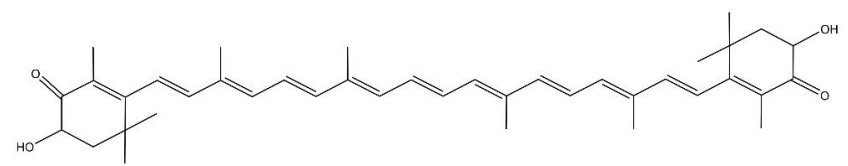

Figure 1 The structure of astaxanthin.

molecular polarity is low, the water solubility is poor, and the bioavailability is low.

The liquid system is converted to a solid state by a simple physical mixing using a solid absorbent carrier. Through the analysis of the fluidity and the adsorption capacity of the powder, silica and anhydrous calcium hydrogen phosphate are selected as the solid carrier of the liquid self-emulsifying system, and the astaxanthin is wrapped in the solid carrier. The result shows that the above $40 \%$ of astaxanthin is released from the solid self-emulsifying system within $120 \mathrm{~min}$. After digestion, the bioavailability can be up to $10 \%$. The results of in vitro dissolution and digestion show that the prawns are delayed release after solid adsorption. ${ }^{[10]}$

Shanmugapriya et al. prepared astaxanthin and tocopherol nanoparticles by spontaneous emulsification and ultrasound with sodium caseinate as raw material. The results showed that the nanoparticles had high stability, small particle size, spherical droplets, unique Zeta potential, non-toxicity and fast cell migration at normal $\mathrm{pH}$ value. It can maintain the protective effect of intracellular reactive oxygen species (ROS), oxidative stress and mitochondrial membrane potential, inhibit cell death through apoptosis mediated by mitochondria, reduce the apoptotic morphology of cancer cells, and initiate cell survival to a greater extent. Thus, it showed significant therapeutic effect in the field of tumor. ${ }^{[11]}$ Shen et al. studied the effects of different emulsifiers (whey protein isolate, poly whey protein, Tween 20, etc.) on the physical and chemical properties and stability of astaxanthin emulsion. ${ }^{[12]}$ The results showed that the droplet size of the emulsion was between 19.4 and $287 \mathrm{~nm}$. The entrapment efficiency of astaxanthin is as high as $90 \%$. At $4{ }^{\circ} \mathrm{C}$, astaxanthin loaded emulsion showed good physicochemical stability. There was a significant effect on the rate of the degradation of astaxanthin, and the uptake of astaxanthin in the emulsion was significantly higher than that of free astaxanthin. Whey protein emulsion has the potential to improve the absorption of astaxanthin cells. Natural banana starch was isolated by Chen et al.. ${ }^{[13]}$ which was modified to resistant starch (type III) by autoclaved cooling process and the steamed banana starch (LABS). The polysaccharide-protein conjugate was prepared by using the soybean protein isolate and used as the wall material of the stable oil-in-water emulsion system to improve the stability of the astaxanthin. The results showed that the astaxanthin showed better stability in the environment of $(6$, $20,37^{\circ} \mathrm{C}$ ).

\section{Anthraquinones}

Anthraquinone is a kind of important natural and synthetic compound, and it has extensive pharmacological actions including the activity of resisting bacteria, resisting oxidation, relaxing bowels, resisting tumor, etc. ${ }^{[14]}$ However, it is extremely difficult to dissolve in water. Taking emodin as an example, the effect of emodin emulsion on the properties of emodin was introduced.

Emodin is a dihydroxyl-like derivative (Figure 2), and has various pharmacological activities such as anti-inflammatory, antibacterial, antiviral, antioxidant, liver-protecting, anti-tumor, and so on ${ }^{[15,16]}$ However, emodin is hardly soluble in water and is not easy to absorb in the body, and its bioavailability is low.<smiles>Cc1cc(O)c2c(c1)C(=O)c1cc(O)cc(O)c1C2=O</smiles>

Figure 2 The structure of emodin.

Huang et al. obtained the best formula of emodin self-microemulsion ${ }^{[17]}$ through solubility study, compatibility test, pseudoternary phase diagram analysis and central composite design. The average droplet size of the self-microemulsion is about $18.31 \pm 0.12 \mathrm{~nm}$ at different dilution multiples, different $\mathrm{pH}$ values (1.2-7.2). The droplet size and zeta potential remain stable. Moreover, the area under curve (AUC) value of emodin self-micro- emulsion was 1.87 times larger than that of emodin suspension, which improved oral utilization of emodin. Zhang et al. ${ }^{[18]}$ prepared emodin nanoemulsion by modified emulsification method and characterized its particle size, morphology, stability and drug release. The emulsion with particle size of $116 \pm 6.5 \mathrm{~nm}$ was obtained. Ma et al. ${ }^{[19]}$ combined self-emulsion technology with pellets technology to solve the problems of low solubility of emodin and poor stability of self-emulsion. Taking the cinnamon oil as an oil phase, taking the RH40 : Tween-80 1:1 as an emulsifier, the auxiliary emulsifier is 1,2-propylene glycol, and the hydroxypropyl methyl cellulose $\mathrm{K} 4 \mathrm{M}$ is a solid carrier to prepare the emodin self-micro-emulsion-pellet. The cumulative release rate of emodin in 20 min was more than $90 \%$. The dissolution behavior of emodin was similar and dispersed in pellets in an amorphous state.

\section{Diketone Compounds}

Turmeric is dried rhizome of turmeric (curcumalonga I.), native to India and now widely cultivated in tropical and subtropical regions including China. It is a perennial herb with medicinal and food homology. Aromatic yellow powder from mature rhizomes, was often used as food additives. ${ }^{[20]}$ Curcumin is a rare pigment with diketones in the botanical world and is a diketone compound (Figure 3 ).<smiles>COc1ccc(/C=C/C(=O)CC(=O)/C=C/c2ccc(OC)c(O)c2)cc1O</smiles>

Figure 3 The structure of curcumin.

The liquid micro-emulsion of curcumin is encapsulated in the micro-pellets by using the ability of the sodium alginate to wrap the medicine, so that the research on the solid-state of the liquid micro-emulsion is realized, and the sodium alginate micro-pill has an effect of slow-release and controlled-release. When combined with the liquid micro-emulsion, the characteristic of the micro-emulsion is not changed. ${ }^{[21]}$ The curcumin nano-emulsion is prepared by using medium-chain fatty acid (MCT), polyethylene glycol-400 and polyoxyethylene and hemp oil. The prepared emulsion has a particle size of less than $20 \mathrm{~nm}$, and the sample has good stability in two weeks. The results show that the curcumin self-microemulsion drug delivery system can release more than $90 \%$ of the drug in the 
dissolution medium containing sodium dodecyl sulfate (SDS), and the release of the curcumin powder in the same medium is less than $40 \% .{ }^{[22]}$ Pan et al. prepared octenyl succinic anhydride (OSA) modified dextrin and characterized it as a new emulsifier to improve the stability of emulsion and encapsulation of curcumin. After the modification of OSA, the emulsion stability of the emulsion is greatly improved. In the process of storage, the particle size of the emulsion is obviously reduced with the increase of the addition amount of the OSA. The modified dextrin of the OSA can improve the encapsulation efficiency of the curcumin. ${ }^{[23]} \mathrm{Xu}$ et al. studied the effect of phytic acid on the oil-in-water nanofat digest and curcumin bioavailability using the simulated gastrointestinal tract. With the increase of phytic acid level, the total amount of free fatty acids released decreased significantly, and the bioavailability of curcumin increased significantly from $39.4 \pm 3.5 \%$ (control group) to $74.7 \pm 2.6 \%\left(0.5 \%\right.$ phytic acid). ${ }^{[24]}$ Liu et al. improved the thermal stability of curcumin pickling emulsion using whey protein isolate (WPI), lactose (Lac) Maillard reaction product and Gallic catechin gallate (EGCG) complex. The analysis of microstructure and droplet size distribution showed that compared with WPI, the saccharified WPI-lac/egcg stable emulsion had more uniform droplet distribution, stronger thermal stability and higher curcumin retention rate. ${ }^{[25]}$

\section{Flavonoids}

The flavonoid compounds have various biological activities such as oxidation resistance, anti-virus, anti-tumor and scavenging free radicals, but the bioavailability is low due to poor solubility and poor stability of the flavonoid compounds. ${ }^{[26]}$

\section{Nobiletin}

As the main active substance in Traditional Chinese Medicine, tangerine peel has a variety of biological activities, including antioxidant, anti-tumor, anti-inflammatory, anti-cancer and immune-regulating activities (Figure 4). ${ }^{[27,28]}$<smiles>COc1ccc(-c2cc(=O)c3c(OC)c(OC)c(OC)c(OC)c3o2)cc1OC</smiles>

Figure 4 The structure of nobiletin.

Shu et al. prepared cinnamaldehyde/chitosan microspheres by chitosan and emulsification technology, which were used to contain nobiletin. When the content of nobiletin was high, it was beneficial to the formation of microspheres and the appearance was more regular. In vitro release test showed that the release rate of nobiletin was slower when the content of cinnamaldehyde was high, and the release of nobiletin could be better controlled by microspheres treated at room temperature or heat treatment. The results of simulated digestion in vitro showed that the bioavailability of nobiletin in emulsion was $60.8 \%$, that in cinnamaldehyde/chi- tosan microspheres treated at room temperature was $48.3 \%$, and that in heat treated cinnamaldehyde/chitosan microspheres was $33.3 \%$. Cinnamaldehyde/chitosan microspheres containing nobiletin could effectively control the release of nobiletin in gastrointestinal tract. ${ }^{[29]} \mathrm{An}^{[30]}$ used high polymer hydroxypropyl methyl cellulose (HPMC) and heat treatment to inhibit the crystallization of persaturated chlorhexidine from nanoemulsion. The effect of the different nano-emulsion system and the stable state of the nobiletin on the bioavailability of the nobiletin was discussed by in-vitro digestion experiment. The results show that the heat treatment can not only change the particle size and appearance of the emulsion, but also greatly improve the encapsulation stability of nobiletin in the emulsion. When the initial supersaturation concentration is high, the bioavailability of HPMC is not obvious, but the bioavailability after heat treatment is $57.84 \%$ higher than that before heat treatment. Sun et al. also used HPMC to prepare the emulsion of nobiletin, and further modified its structure by changing the oil (glycerol monolaurate, GML) and water phase (whey protein WPC). On this basis, the encapsulation and release of nobiletin were evaluated. When $3 \%$ HPMC was added, the particle size of oil droplets was stabilized at $370 \mathrm{~nm}$. When HPMC was used as emulsifier, the stabilization time of nobiletin in emulsion was prolonged and the encapsulation amount was about $4.5 \mathrm{mg} / \mathrm{g}$. The addition of GML and WPC changes the microstructure of the stable emulsion of HPMC, and after the modification, the amount of the encapsulating agent is $7.5 \mathrm{mg} / \mathrm{g}$, and it can be stably placed for one week. In the presence of GML and WPC the biological availability of nobiletin can be adjusted. The results show that the structure of the emulsifier system influences the encapsulation and transfer of the hydrophobic component. ${ }^{[31]}$ Liao et al. mixed nobiletin into oil phase and prepared nano-emulsion by high speed and high pressure homogenization method using medium chain triacylglycerol as oil phase. The mean particle size of nobiletin nanoemulsion was $168.6 \pm 3.8 \mathrm{~nm}$ and PDI was 0.168 , while the mean particle size of blank nanoemulsion was $157.3 \pm 1.9 \mathrm{~nm}$ and PDI was 0.161 . The zeta potential of nobiletin nanoemulsion and blank nanoemulsion was $-68.45 \pm 0.64 \mathrm{mV}$ and $-62.75 \pm 0.21 \mathrm{mV}$, respectively. All the obtained nanoemulsions maintained physical stability during storage at 4,25 and $37^{\circ} \mathrm{C} .^{[32]}$

\section{Quercetin}

Quercetin is a polyhydroxy flavonoid compound (Figure 5), and it is present in a variety of fruits, vegetables and plants, and has the functions of resisting oxidation, reducing blood sugar, resisting cancer, and resisting inflammation. The low bioavailability restricts the application of quercetin. ${ }^{[33,34]}$<smiles>O=c1c(O)c(-c2ccc(O)c(O)c2)oc2cc(O)cc(O)c12</smiles>

Figure 5 The structure of quercetin.

Son et al. prepared quercetin nanoemulsion (NQ) and investigated the effect of $\mathrm{pH}$ on the formation of $\mathrm{NQ}$. The results showed that the encapsulation rate increased from $56 \%$ to $92 \%$ during the $\mathrm{pH}$ increase from 4.0 to 9.0 . In the $\mathrm{pH}$ range of $6.5-9, \mathrm{NQ}$ was stored for $3 \mathrm{~m}$ at 21 and $37{ }^{\circ} \mathrm{C}$ with good stability. ${ }^{[35]}$ Compared with quercetin, NQ was more effective in reducing serum liver cholesterol levels and increasing the release of bile acids into feces in rats fed a high-cholesterol diet. Chen et al. prepared quercetin emulsions to improve the biological availability of quercetin, a hydrophobic nutrient Sodium caseinate, whey protein isolates or soy protein isolates, were used as emulsifiers to prepare water-in-oil emulsions of protein stability. Then the emulsions were mixed with quercetin powder and heated to simulate the cooking process. The 
results show that the adsorption ability of casein to the oil-water interface is strong, and it has a more stable interface layer than the other two proteins. There were appreciable differences in quercetin bioaccessibility in excipient emulsions stabilized by different emulsifiers $(\approx 74 \%$ for casein, $54 \%$ for whey protein, $22 \%$ for soy protein, and $58 \%$ for Tween). It is proved that the milk protein may be a natural alternative to the synthetic surfactant, which can form a stable emulsion and improve the biological availability of the nutrients. ${ }^{[36]}$

Based on palm oil ester (POE), Arbain et al. ${ }^{[37]}$ prepared quercetin lung-administered nanoemulsion. Nano-emulsion was prepared by high energy emulsion method and optimized by $D$-optimal mixing design. The results showed that ricinoleic acid (RC), lecithin, Tween 80 , glycerin and $93.90 \%$ water were used as the main raw materials, and $1.5 \mathrm{wt} \%$ POE was added to obtain the average particle diameter of $110.3 \mathrm{~nm}$ nanoemulsion. The PDI and Zeta potential were 0.290 and 37.7 $\mathrm{mV}$, respectively. The preparation also showed good stability when stored at $4{ }^{\circ} \mathrm{C}$ for $90 \mathrm{~d}$. Moreover, the POE nanoemulsion containing quercetin had good physical properties and atomization efficiency, and had the potential to be used in the treatment of lung cancer.

\section{Baicalein}

Scutellaria baicalensis, a traditional Chinese herbal medicine, is mainly used to dissipate phlegm, clear heat, moisten, detoxify, stop bleeding and prevent miscarriage, etc. ${ }^{[38]}$ Baicalein has the main functions of antibacterial, antiviral, anti-inflammatory, antioxidant, anti-tumor, free radical scavenging, lipid lowering, immune regulation, etc (Figure 6). ${ }^{[39]}$<smiles>O=c1cc(-c2ccccc2)oc2cc(O)c(O)c(O)c12</smiles>

Figure 6 The structure of baicalein.

Baicalein (BCL) has high pharmacological activity, but its solubility and stability in intestinal tract are poor. Yin et al. discussed the potential of nano-emulsion composed of hemp oil and a small amount of surfactants to improve the oral bioavailability of BCL. The loaded BCL meter composites were prepared by high pressure homogenization method. The average particle diameter of $90 \mathrm{~nm}$ nano-emulsion was obtained. BCL nano-emulsion significantly improved the oral bioavailability of $\mathrm{BCL}$. Compared with traditional emulsion and suspension, BCL nano-emulsion increased by $524.7 \%$ and $242.1 \%,{ }^{[40]}$ respectively. Meng et al. prepared paclitaxel/ baicalin nanometer emulsion (PTX/BA NE) to overcome multidrug resistance (MDR) in breast cancer. More importantly, in vivo anti-tumor studies have shown that PTX/BA NE has higher anti-tumor efficacy than other PTX preparations. ${ }^{[40]}$ Nipun et al. prepared chitosan (CS) nanoparticles and baicalin-loaded chitosan nanoparticles in W/O emulsion system using cinnamaldehyde as cross-linking agent. Its load efficiency and packaging efficiency are $9.1 \%$ and $97.2 \%$, respectively. ${ }^{[41]}$

\section{Biochanin A}

Generation pharmacological studies have shown that chickpea extracts, especially chickpea isoflavones and saponins, have hypoglycemic, lipid-lowering, cholesterollowering, anti-aging, anti-oxidation, anti-tumor, insect repellent and other pharmacological activities. ${ }^{[42]}$ Biochanin $A$ is a natural organic compound (Figure 7), belonging to the flavonoid phytochemicals of trifolium pratense, with a wide range of biological activities, such as anticancer, antifungal, antitumor, antiviral, anti-inflammatory, antioxidant and antidiabetic activities. $^{[43-45]}$<smiles>COc1ccc(-c2coc3cc(O)cc(O)c3c2=O)cc1</smiles>

Figure 7 The structure of Biochanin A.

Wang et al. prepared biochickanin A (BCA) lipid nanoparticles by emulsion evaporation and low temperature solidification. The results showed that the average particle size $(148.5 \pm 2.88 \mathrm{~nm})$ of the BCA-PEG-NCL was small. The results revealed that BCA-PEG-NLC had small mean particle $(148.5 \pm 2.88 \mathrm{~nm})$ with narrow polydispersity index $(\mathrm{PI})$ $(0.153 \pm 0.01)$, encapsulation capacity $(99.62 \pm 0.06 \%)$, payload $(9.06 \pm 0.01 \%)$, zeta potential $(-19.83 \pm 1.19 \mathrm{mV})$. Especially in pharmacokinetics, the Cmax value and the area under curve (AUC) of bca-peg-nlc were higher than that of BCA suspension (about 15.8 times and 2.9 times, respectively), while the mean residence time (MRT) was significantly longer. ${ }^{[46]}$ In order to improve the efficiency of intradermic administration of genistein and two other isoflavones (daidzein and biochanin A), Kitagawa et al. used isopropyl nutmeg (IPM), $150 \mathrm{mM} \mathrm{NaCl}$ solution, Tween 80 and ethanol microemulsion as carrier to significantly improve the solubility of isoflavones and introduce a large number of isoflavones into the skin. ${ }^{[47]}$

\section{Conclusion}

In view of the unique physiological functions of insoluble substances, the market potential for the development of related food, functional food, medicine and daily chemical products is huge. In recent years, the design of delivery system to improve the above shortcomings has aroused great interest of researchers in various countries.

The research on emulsion delivery system of insoluble substances in China is still in its initial stage, and many problems need to be further studied. For example, the combination of emulsion delivery system and other carrier systems, the changes of stability and interfacial properties of emulsion carrier system in digestive tract, and the mechanism of its influence on digestion, absorption and metabolism, the targeted release of insoluble active substances in digestive tract and the targeted release of targeted tissue in vivo, and so on. The research and solution of the above problems not only depends on the progress of technology, but also requires the interaction between disciplines, which will be beneficial to the improvement of the research technology and depth of insoluble active material delivery system and promote the development of insoluble active material industry.

\section{Conflict of Interest}

The authors declare no conflict of interest.

Copyright $\odot 2019$ Xueyan Li, Junwen Wang, and Chaoyan Zhang. This article is an open access article distributed under the terms and 
conditions of the Creative Commons Attribution (CC BY) license (http://creativecommons.org/licenses/by/4.0/). The use, distribution or reproduction in other forums is permitted, provided the original author(s) or licensor are credited and that the original publication in this journal is cited, in accordance with accepted academic practice. No use, distribution or reproduction is permitted which does not comply with these terms.

\section{References}

[1] Cerpnjak, K.; Zvonar, A.; Gašperlin, M.; Vrečer, F. Acta Pharmaceut. 2013, 427, 445.

[2] Kotta, S.; Khan, A. W.; Pramod, K. Expert Opin. Drug Del. 2018, $585,598$.

[3] Christofori, N.; Thellie, P.; Eman, A. Pharmaceutics 2017, 37.

[4] Patel, R. D.; Thakore, S. D.; Patel, M. R. Curr. Drug Deliv. 2016, $857,81$.

[5] Lawrence, M. J.; Rees, G. D. Adv. Drug Deliver. Rev. 2012, 175, 193.

[6] Mundada, V.; Patel, M.; Sawant, K. Crit. Rev. Ther. Drug Carrier Systems 2016, 265, 308.

[7] Morais, A. R.; Alencar, Édo, N. Int. J. Pharmaceut. 2016, 102, 114.

[8] Honda, M.; Kageyama, H.; Hibino, T. Molecules 2019, 24, 2149.

[9] Delgado, A. M. A.; Khandual, S. I.; Villanueva, S. Food Chem. 2017, 23, 30.

[10] Mao, X.; Sun, R.; Tian, Y. Eur. J. Lipid Sci. Technol. 2019, 1800258.

[11] Khan, I.; Bahuguna, A.; Kumar, P. Sci. Rep. 2018, 144.

[12] Shen, X.; Fang, T.; Zheng, J. Molecules 2019, 24, 727.

[13] Chen, Z.; Shu, G.; Taarji, N. Food Chem. 2018, 322, 328.

[14] Xun, L.; Liu, Y.; Chu, S. Chem. Biol. Interact 2019, 108722.

[15] Liu, Y.; Dong, X. X.; Ni, J. China J. Trad. Chin. Med. Pharm. 2019, 2028, 2034 (in Chinese).

[16] Xiong, H. R.; Luo, J.; Hou, W. J. Eth-nopharmacol. 2011, 723.

[17] Huang, J.; Gong, W.; Chen, Z. Eur. J. Pharm. Sci. 2017, 128, 136.

[18] Zhang, T.; Dong, D.; Lu, D. Int. J. Pharm. 2016, 190, 98.

[19] Ma, Z. B.; Li, X. F.; Xie, L. J. Chin. Med. Mater. 2019, 144, 149 (in Chinese).

[20] Huang, Y. B. D. Dissertation, Chongqing Medical University, Chongqing, China, 2013 (in Chinese).

[21] Sun, L. L.; Qiao, L.; Tian, Z. H. Journal of Shandong University of Traditional Chinese Medicine 2019, 207, 212 (in Chinese).
[22] Li, Z. M.; Li, X. Q.; Xu, X. H. Journal of Chengdu Medical College 2017, 44, 48 (in Chinese).

[23] Pan, Y.; Wu, Z.; Zhang, B. Food Chem. 2019, 326, 332.

[24] Xu, W.; Huang, L.; Jin, W. Int. J. Biol. Macromol. 2019, 210, 215

[25] Liu, G.; Wang, Q.; Hu, Z. J. Agric. Food Chem. 2019, 5212.

[26] Xiao, Y. M.; Li, M.; Yuan, Y. W. Journal of Henan University of Technology 2019, 123, 131 (in Chinese).

[27] Umeno, A.; Horie, M.; Murotomi, K. Molecules 2016, 21, 708.

[28] Qi,Y. L.; Li, S. Z.; Zhu, D. L. Journal of Harbin Medical University 2019, 35, 38 (in Chinese).

[29] Shu, Q.; Feng, H. Journal of Central China Normal University (Natural Sciences) 2018, 804, 810 (in Chinese).

[30] An, Y. P. B.D. Dissertation, Huazhong Agricultural University, Wuhan, China, 2015 (in Chinese).

[31] Sun, G.; Lei, L.; Chen, H. Food Funct. 2018, 3657, 3664

[32] Liao, W.; Liu, Z.; Zhang, T. J. Agr. Food Chem. 2018, 91, 98

[33] Li, Y.; Yao, J.; Han, C. Nutrients 2016, 167.

[34] Sun, H.; Jin, H.;Yang, R. R. Farm Prod. Process. 2019, 84, 88 (in Chinese).

[35] Son, H. Y.; Lee, M.S. Nutrients 2019, 11, 244.

[36] Chen, X.; McClements, D. J.; Zhu, Y.; Chen, Y.; Zou, L.; Liu, W.; Cheng, C.; Fu, D.; Liu, C. Food Res. Int. 2018, 30, 37.

[37] Arbain N. H.; Salim, N.; Wui W. T. J Oleo Sci. 2018, 933.

[38] Jiang, M.; Li, Z. N.; Zhu, G. X. Complement. Ther. Med. 2018, 11.

[39] Han, H. China Science and Technology Information 2019, 32, 34 (in Chinese).

[40] Yin, J.; Xiang, C.; Wang, P. Int. J. Nanomed. 2017, 2923.

[41] Meng, L.; Xia, X.; Yang, Y. Int. J. Pharm. 2016, 8, 16.

[42] Babu, V. N.; Kannan, S. Int. J. Biol. Macromol. 2012, 1103, 8.

[43] Shi, X. J. B. D. Dissertation, Bei Jing University of Chinese Medicine, Beijing, China, 2016 (in Chinese).

[44] Harni, R.; Ezhumalai, M.; Kodukkur, V. P. Eur. J. Pharmacol. 2012, 89, 94.

[45] Puli, S, Lai J. C. Bhushan, A. J. Neuro-Oncol. 2006, 135, 42.

[46] Kole, L.; Giri, B. Manna, S. K. Eur. J. Pharmacol. 2011, 8, 15.

[47] Wang, L.; Luo, Q.; Lin, T. Drug Dev. Ind. Pharm. 2015, 1204, 1212.

[48] Kitagawa, S.; Inoue, K; Teraoka, R. Chem. Pharm. Bull. 2010, 398, 401. 